\title{
Accelerated C-N Bond Formation in Dropcast Thin Films on Ambient Surfaces
}

\author{
Abraham K. Badu-Tawiah, Dahlia I. Campbell, R. Graham Cooks \\ Department of Chemistry, Purdue University, West Lafayette, IN 47907, USA
}

\begin{abstract}
The aza-Michael addition and the Mannich condensation occur in thin films deposited on ambient surfaces. The reagents for both $\mathrm{C}-\mathrm{N}$ bond formation reactions were transferred onto the surface by drop-casting using a micropipette. The surface reactions were found to be much more efficient than the corresponding bulk solution-phase reactions performed on the same scale in the same acetonitrile solvent. The increase in rate of product formation in the thin film is attributed to solvent evaporation in the open air which results in reagent concentration and produces rate acceleration similar to that seen in evaporating droplets in desorption electrospray ionization. This thin film procedure has potential for the rapid synthesis of reaction products on a small scale, as well as allowing rapid derivatization of analytes to produce forms that are easily ionized by electrospray ionization. Analysis of the derivatized sample directly from the reaction surface through the use of desorption electrospray ionization is also demonstrated.
\end{abstract}

Key words: $\mathrm{C}-\mathrm{N}$ bond formation, Aza-Michael addition, Mannich reaction, Surface chemistry, Solvent effects, Ion chemistry, Microdroplets, Mass spectrometry

\section{Introduction}

$\mathrm{T}^{\mathrm{s}}$ he formation of carbon-nitrogen $(\mathrm{C}-\mathrm{N})$ single bonds is important in diverse areas of chemistry because of the ubiquitous presence of nitrogen-containing compounds in nature. For example, the formation of $\mathrm{C}-\mathrm{N}$ bonds plays a significant role in the synthesis of pharmacologically active molecules as well being essential in such key biological processes as peptide/protein synthesis. We have an ongoing interest in chemical reactions in charged microdroplets [1], which is extended here to the study of reactions in thin films. Aza-Michael addition (Scheme 1a) is a direct approach to the construction of $\mathrm{C}-\mathrm{N}$ bonds. It represents a valuable synthetic alternative to the established Mannich reaction (Scheme 1b). Under bulk solution-phase conditions, the azaMichael addition is catalyzed by Lewis acids [2,3] (under

Electronic supplementary material The online version of this article (doi:10.1007/s13361-012-0394-y) contains supplementary material, which is available to authorized users.

Correspondence to: R. G. Cooks; e-mail: cooks@purdue.edu strictly anhydrous conditions), metal catalysts [4-6] [e.g., $\left.\mathrm{Yb}(\mathrm{OTf})_{3}, \mathrm{InCl}_{3}, \mathrm{FeCl}_{3} .7 \mathrm{H}_{2} \mathrm{O} / \mathrm{CO}(\mathrm{OAc})_{2}\right]$, oxidizing agents [7,8] (e.g., ceric ammonium nitrate) or $N$-heterocyclic carbenes [9] generated by treating imidazolium salts with a base). In this study, interfacial versions of the aza-Michael addition and Mannich reactions were carried out under ambient conditions in high yields (on a small scale) without the use of catalyst. The synthetic procedure involves simple drop-casting of the reagents onto a surface in the open air and reaction is thought to be driven by reagent concentration via solvent evaporation.

Solvent evaporation leading to rate enhancement of chemical reactions has recently been reported for charged microdroplets reactor systems generated in reactive desorption electrospray ionization (DESI) $[1,10,11]$ and ambient ion soft landing [12-14] experiments. Traditionally, the advantages of microreactor systems are recognized to include rapid mixing of reagents, effective control of reaction time, and the ability to control interfacial chemistry [15]. It has also become apparent that the larger surface areas associated with microreactor systems favor physical processes such as evaporation (when this is allowed) and 
(a)

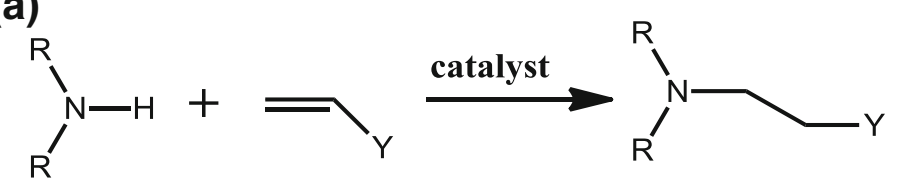

(b)

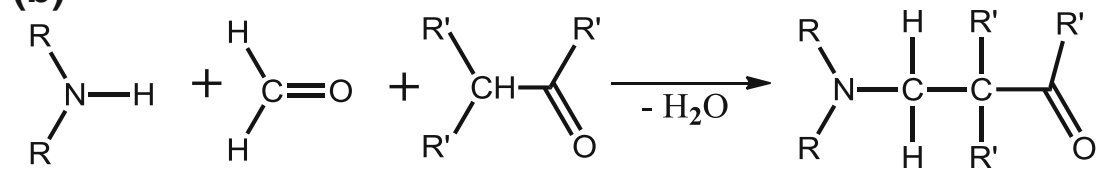

Scheme 1. (a) Aza-Michael addition; $\mathrm{Y}=\mathrm{CONH}_{2}, \mathrm{CN}, \mathrm{COMe}, \mathrm{CO}_{2} \mathrm{Et}(\mathrm{Me})$, and (b) general reaction scheme depicting the Mannich reaction

partitioning of solutes from the bulk to the surface [16-18], which should increase those reactions that are dependent on surface active species. In the special case of charged microdroplets, the increase in product yield is ascribed to solvent evaporation, which causes moderate $\mathrm{pH}$ values in the starting droplet to reach extreme values while reagent concentrations also increase [1,19]. Such a system might be particularly suitable for accelerating acid/base catalyzed bimolecular reactions. Aside from microreactors, solid films of reactants containing microscopic quantities of solvent have been reported to serve as efficient reaction media in which organic reactions can be accelerated [12,20]. Otera and coworkers generated solid films through the use of a rotary evaporator [20], and reaction was observed to be more efficient than that under the corresponding bulk solutionphase conditions when the solid film was allowed to stand under ambient conditions. This procedure was applied to imine synthesis, the Wittig reaction, and quaternization of tertiary phosphine, and pyridine [20]. Gentle deposition of electrospray [21,22] droplets at ambient surfaces has been shown to afford organic solid films in which the Girard condensation is accelerated compared with the bulk solution-phase reaction conducted on the same scale [12]. In this paper, we show that similar enhancement in product yield can be achieved for $\mathrm{C}-\mathrm{N}$ bond forming reactions in nominally dry mixtures generated by simple drop-casting of reactant solutions onto ambient surfaces. The drop-casted products are easily collected or directly analyzed using surface analysis techniques such as DESI without dissolution.

\section{Experimental}

\section{Chemicals and Reagents}

Acrylamide, acrylonitrile, methyl acrylate, morpholine, piperidine, cycloheptylamine, benzaldehyde, cyclohexanone, and chloroform were purchased from Sigma-Aldrich (Milwaukee, WI, USA), ethanolamine was obtained from Acros Organics (Geel, Belgium), and methanol and acetonitrile (HPLC grade) from Mallinckrodt Baker Inc. (Phillipsburg, NJ, USA). Deionized/distilled water was obtained using a
Barnstead/Thermolyne deionizer unit (Barnstead Mega-Pure System, Dubuque, IA, USA).

(a)
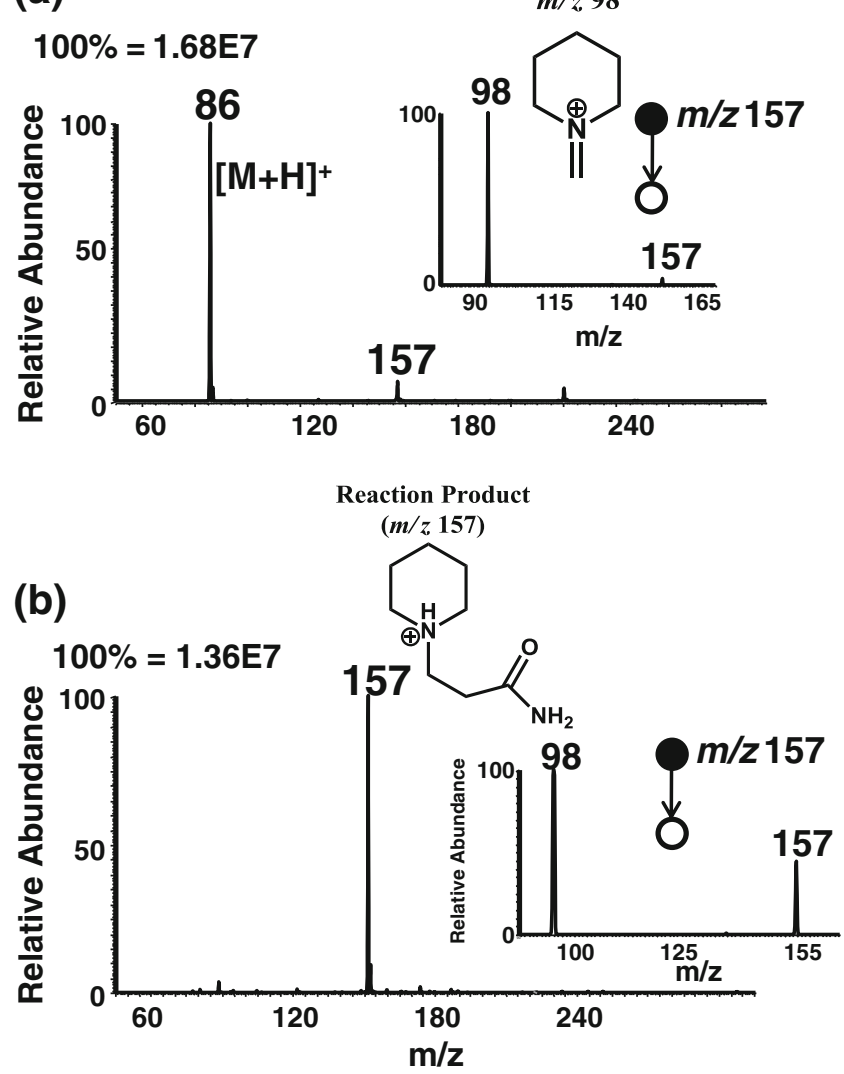

Figure 1. Nanospray-MS of piperidine (M) $(33 \mathrm{mM})$ and acrylamide $(28 \mathrm{mM})$ reaction mixture after (a) $1 \mathrm{~h}$ bulk solution-phase reaction, and (b) 10 min surface reaction. In both cases 2, $\mu \mathrm{L}$ of each reagent in acetonitrile was used without catalyst. After $10 \mathrm{~min}$ reaction time, the surface reaction mixture was dissolved in $10 \mu \mathrm{L}$ of methanol/water $(1: 1, \mathrm{vol} / \mathrm{vol})$ and analyzed using nanospray ionization $(1.8 \mathrm{kV})$. Similarly, after $1 \mathrm{~h}$ bulk-phase reaction, $10 \mu \mathrm{L}$ of methanol/water $(1: 1, \mathrm{vol} / \mathrm{vol})$ was added to the reaction mixture for MS analysis. Inserts indicate MS/MS product ion spectrum of reaction product at $\mathrm{m} / \mathrm{z} 157$ 


\section{Nanospray Ionization Mass Spectrometry for Product Analysis}

Surface reaction was achieved by depositing appropriate volumes (typically $2 \mu \mathrm{L}$ ) of each of the reagent solutions to be reacted separately (but at the same spot) onto a surface, and allowing the resulting drop to dry under ambient conditions. After surface reaction, the dried material was dissolved into $10 \mu \mathrm{L}$ of methanol/ water $(1: 1, \mathrm{vol} / \mathrm{vol})$ and characterized using nanoelectrospray ionization-MS (nanospray-MS or nESI-MS). A nanospray was generated using an emitter (E Series Microelectrode Holder, with Ag wire electrode; Warner Instruments, LLC, Hamden, CT, USA) and applying a voltage of $1.8 \mathrm{kV}(\sim 20 \mathrm{~nL} / \mathrm{min}$ flow rate). These sample analysis conditions involve short ionization periods, and so significant reaction cannot occur during analysis. All MS experiments were performed using a Thermo Fisher Scientific LTQ mass spectrometer (San Jose, CA, USA). Typical MS parameters used included averaging of 3 microscans, $100 \mathrm{~ms}$ maximum ion injection time, $15 \mathrm{~V}$ capillary voltages, $150{ }^{\circ} \mathrm{C}$ capillary temperature, and $65 \mathrm{~V}$ tube lens voltage. Data were acquired and processed using Xcalibur 2.0 software (Thermo Fisher Scientific, San Jose, CA, USA). The identification of analyte ions was confirmed by tandem mass spectrometry using collision-induced dissociation (CID). An isolation window of $1.5 \mathrm{Th}$ (mass/charge units) and a normalized collision energy of 30 \%-35 \% (manufacturer's unit) was selected for the CID experiments. Reagent (e.g., piperidine) consumption was calculated based on normalized product ion intensities $\left[\mathrm{I}_{\mathrm{P}} /\left(\mathrm{I}_{\mathrm{P}}+\mathrm{I}_{\mathrm{R}}\right)\right]$, where $\mathrm{I}_{\mathrm{P}}$ and $I_{R}$ represent protonated product and reagent ion intensities, respectively.

Table 1. Acrylamide Reaction with Amines (in Acetonitrile Solution) at Ambient Surface in the Open Air

\begin{tabular}{|c|c|c|c|c|c|}
\hline \multirow[b]{2}{*}{$\begin{array}{l}\text { Reagent } \\
\text { name }\end{array}$} & \multirow[b]{2}{*}{$\begin{array}{l}\text { Reagent } \\
\text { structure }\end{array}$} & \multirow[b]{2}{*}{$\begin{array}{l}\text { Reaction } \\
\text { product }\end{array}$} & \multirow[b]{2}{*}{$\begin{array}{l}\text { Surface } \\
\text { reaction Time } \\
\text { (min.) }\end{array}$} & \multicolumn{2}{|c|}{$\%$ Reagent consumed $^{a}$} \\
\hline & & & & $\begin{array}{l}\text { Reaction at } \\
\text { surface }\end{array}$ & $\begin{array}{c}1 \mathrm{~h} \\
\text { bulk-phase } \\
\text { reaction }\end{array}$ \\
\hline Morpholine & & & 10 & 99 & 16 \\
\hline Piperidine & & & 10 & 99 & 5 \\
\hline Ethanolamine & & & 10 & $99^{b}$ & 92 \\
\hline $\begin{array}{l}\text { Cycloheptyl- } \\
\text { amine }\end{array}$ & & & 20 & $87^{c}$ & 5 \\
\hline & & & 50 & $94^{c}$ & \\
\hline
\end{tabular}

${ }^{a}$ Percent reagent consumption was calculated based on normalized product ion intensities $\left[I_{P} /\left(I_{P}+I_{R}\right)\right]$, where $I_{P}$ and $I_{R}$ represent protonated product and reagent ion intensities, respectively. The same amounts of reactants (acrylamide/amine mole ratio of 1:1.2) were used for the surface reaction as for the bulk solution-phase reaction

${ }^{\mathrm{b}}$ Including $9 \%$ of bis-addition product

${ }^{\mathrm{c}}$ Including $2 \%$ of bis-addition product 


\section{Results and Discussion}

Initial investigations involved the reaction between piperidine (33 $\mathrm{mM})$ and acrylamide $(28 \mathrm{mM})$ using a micropipette to deposit $2 \mu \mathrm{L}$ of each of the reagent solutions in acetonitrile onto a stainless steel surface (each reagent solution was separately deposited at the same spot). About $99 \%$ of piperidine $(6 \mu \mathrm{g}$ absolute, starting amount) was converted into product after $10 \mathrm{~min}$ of surface reaction time, whereas $1 \mathrm{~h}$ of solution-phase reaction in acetonitrile allowed only $5 \%$ piperidine conversion in the absence of a catalyst (Figure 1a) using the same quantities and starting concentrations of reagent as utilized for the surface reaction. Note that the surface products were typically dissolved into methanol/water $(1: 1, \mathrm{vol} / \mathrm{vol})$ and then analyzed using nanospray-mass spectrometry. Comparison of the nESI-MS data for the bulk reaction to that of the surface reaction indicates that the surface products were formed before dissolution and not during MS analysis of the product. The complete list of amines reacted with acrylamide under the dropcasting conditions is provided in Table 1. Note that primary amines such as ethanolamine and cycloheptylamine also yielded bis-addition products by reaction of two molecules of acrylamide instead of one, which was the only reaction in the case of secondary amines. Among all the aliphatic amines tested (Table 1), the reaction involving the sterically hindered cycloheptylamine and acrylamide was found to be exceptionally slow, with a product yield of $94 \%$ in 50 min reaction time compared with $99 \%$ yield for morpholine, piperidine, and ethanolamine in $10 \mathrm{~min}$ of surface reaction with acrylamide.
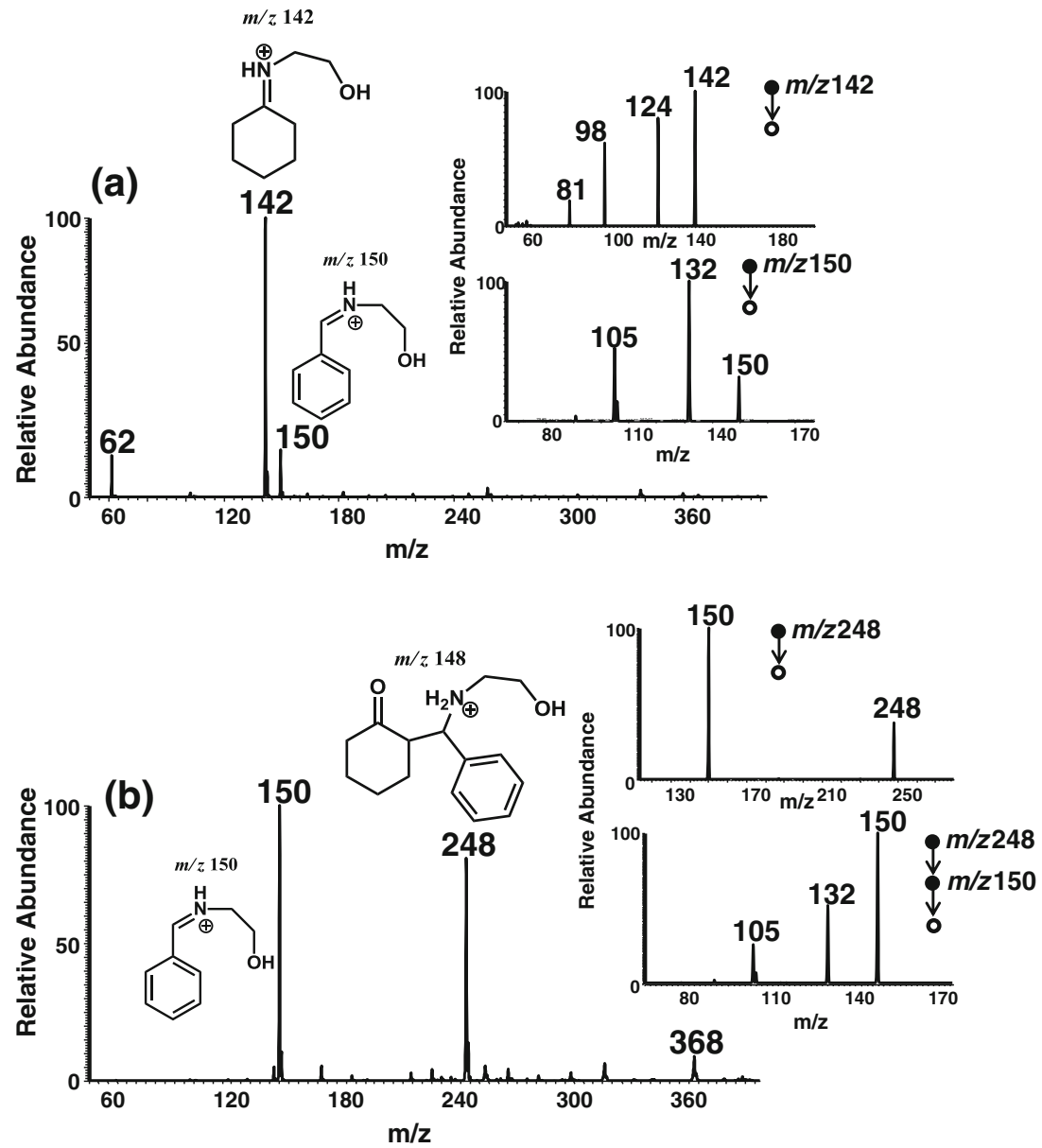

Figure 2. Mannich reaction involving benzaldehyde $(10 \mathrm{mM})$, ethanolamine $(16 \mathrm{mM})$, and cyclohexanone $(0.1 \mathrm{M})$ in acetonitrile (a) under bulk solution-phase and (b) at ambient surface. For the bulk phase reaction, $1 \mu \mathrm{L}$ each of benzaldehyde and ethanolamine was premixed for $1 \mathrm{~h}$ before adding $16 \mu \mathrm{L}$ of cyclohexanone. At the surface, however, the benzaldehyde/ ethanolamine drop ( $1 \mu \mathrm{L}$ each) was allowed to stand in the open air for 2 min before adding $16 \mu \mathrm{L}$ of cyclohexanone. Only 10 min of reaction time was allowed after cyclohexanone was added for bulk-phase reaction after which $10 \mu \mathrm{L}$ of methanol/ water (1:1, vol/vol) was added for MS analysis. Similarly, the surface reaction was allowed an extra 10 min after cyclohexanone was added to the dry benzaldehyde/ethanolamine mixture. The surface reaction mixture was then (re)dissolved into $10 \mu \mathrm{L}$ of methanol/water $(1: 1, \mathrm{vol} / \mathrm{vol})$ and analyzed using nanospray-MS. Inserts indicate MS/MS product ion spectrum of reaction product at $\mathrm{m} / \mathrm{z} 248$, and also for intermediates at $\mathrm{m} / \mathrm{z} 142$ and 150 . The MS product ion spectrum of $\mathrm{m} / \mathrm{z} 248 \mathrm{via} \mathrm{m} / \mathrm{z} 150$ ion is also provided. Ion at $\mathrm{m} / \mathrm{z} 368$ in (b) is an unrelated background ion 
The Mannich reaction (Scheme 1b), which represents another reaction in which $\mathrm{C}-\mathrm{N}$ (and $\mathrm{C}-\mathrm{C}$ ) bonds are formed, was also attempted at ambient surfaces using the dropcasting method. Under bulk solution-phase conditions, this multi-component condensation of a non-enolizable aldehyde, primary or secondary amine, and an enolizable carbonyl compound (i.e., carbonyl compound having one or more $\alpha$-hydrogen atoms) affords $\beta$-amino carbonyl products. Often, the desired Mannich product is generated by indirect procedures in which either the enolate or the imine (i.e., the Schiff base) is preformed [23-25]. Direct one-pot Mannich reactions have been reported, however, catalysts and long reaction times are typically needed $[26,27]$. The thin film approach presented here represents a rapid form of indirect Mannich reaction. No unwanted products are detected as shown by the dissolution of the surface product and analysis via nESI-MS. Specifically, only 2 min of reaction time was needed to generate the desired Schiff base $(\mathrm{m} / \mathrm{z} 150)$ by drop-casting $1 \mu \mathrm{L}$ each of ethanolamine $(16 \mathrm{mM})$ and benzaldehyde $(10 \mathrm{mM})$ solutions from acetonitrile onto a surface. Subsequently, addition of $16 \mu \mathrm{L}$ of cyclohexanone solution $(0.1 \mathrm{M})$ in acetonitrile to the resultant and nominally dry reaction spot on the surface provided the expected Mannich product at $\mathrm{m} / \mathrm{z} 248$ in an extra 10 min (Figure 2b). The formation of this product was confirmed through collision-induced dissociation (CID), as it fragmented to give an ion at $m / z 150$ via the loss of cyclohexanone (MW 98). Note that the intermediate species $(\mathrm{m} / \mathrm{z} 150)$ and the reaction product $(\mathrm{m} / \mathrm{z} 248)$ have different ionization efficiencies so their intensities as represented in the mass spectrum (Figure 2b) do not reflect the relative amounts present in the collected material. However, almost all reactants (ethanolamine and benzaldehyde) were converted into the intermediate before cyclohexanone was added. Under bulk solution-phase conditions, by contrast, $1 \mathrm{~h}$ reaction time involving benzaldehyde and ethanolamine in acetonitrile (the same quantities as used for the surface reaction) did not yield a significant amount of the desired Schiff base needed for reaction with the enolizable carbonyl compound (i.e., cyclohexanone) in the second step. Instead, the subsequent addition of cyclohexanone to this reaction mixture consisting of unreacted ethanolamine and benzaldehyde produced predominantly a second Schiff base at $\mathrm{m} / \mathrm{z}$ 142 via reaction of cyclohexanone with ethanolamine (Figure 2a). Again, CID confirmed the absence of the Mannich product at $m / z 248$.

The general increase in reaction rate observed in the thin films, compared with bulk solution-phase reactions with no added catalyst, is attributed to increased reagent concentration due to solvent and surface effects. This was investigated for the aza-Michael addition by preparing reagents in different solvents (chloroform, acetonitrile, and water) and comparing the yield of the surface reaction as a function of reaction time. The results, shown in Figure 3, indicate that the morpholine reaction at the surface with acrylamide was fastest when the reactants were prepared in chloroform and

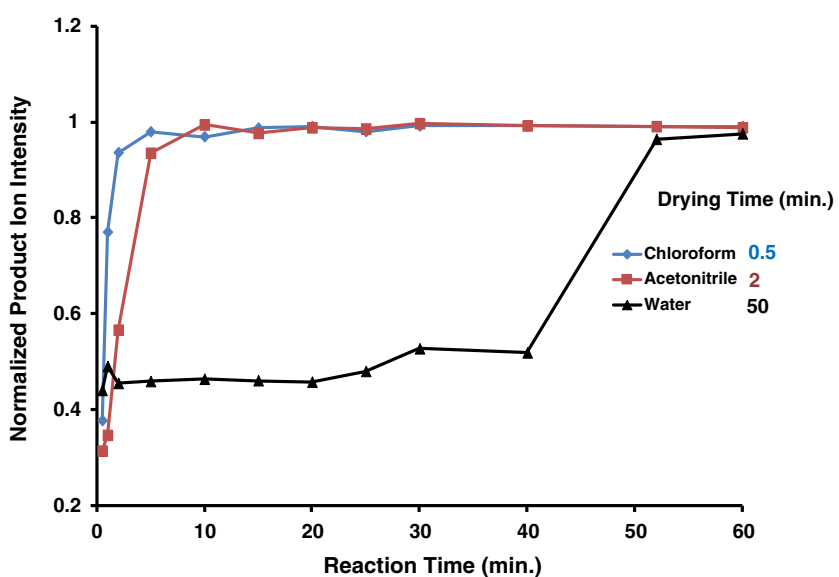

Figure 3. Yield of morpholine reaction with acrylamide (1.2:1 mol ratio) at surface in different solvents. In all cases, $2 \mu \mathrm{L}$ of each reagent in the respective solvents was used without addition of catalyst. Normalized product ion intensity was calculated based on the ratio: $I_{P} /\left[I_{P}+I_{M}\right]$, where $I_{P}$ and $I_{M}$ represent protonated product $(\mathrm{m} / \mathrm{z} 159)$ and morpholine $(\mathrm{m} / \mathrm{z}$ 88) ion intensities respectively

slowest in water. This is simply ascribed to the differences in the rates with which the respective solvents evaporate. For example, it takes $30 \mathrm{~s}$ to generate a nominally dry reaction mixture when chloroform is used as the solvent, compared with 2 and $50 \mathrm{~min}$. for acetonitrile and water, respectively. This indicates that solvent evaporation causes reagent concentration, which leads to more effective collisions. This increased reaction rate attributable to the reduced number of solvent molecules is analogous to the effect of solvent removal on gas-phase $\mathrm{SN}^{2}$ reactions in which essentially no activation energy is required and where every collision results in product formation [28,29]. Michael acceptors having higher vapor pressures such as acrylonitrile and methyl acrylate were found not to be useful in this procedure since solvent evaporation leading to reagent concentration could not be achieved-the solvent and reagents all evaporated together leaving only the amine, with no reaction product at the surface.

The possibility of there being surface effects on reaction efficiency were also investigated. First, chemical effects in the form of possible structural changes of reagent at surfaces due to the absence of solvent were investigated using infrared spectroscopy (Thermo-Nicolet Nexus FTIR, with ATR optics). FTIR spectrum recorded on the nominally dry acrylamide (in the absence of amine) showed a band at about $1670 \mathrm{~cm}^{-1}$, which is attributed to $\mathrm{C}=\mathrm{O}$ stretching of amides [30] (Figure S1, Supporting Information) just as is observed in solution-phase and indicating no structural changes at the surface in the absence of solvent. This result suggests that the increase in surface product yield compared with that obtained under bulk conditions is simply due to solvent evaporation leading to reagent concentration. In this regard, the influence of the physical properties of the surface itself on solvent evaporation was further investigated by utilizing 
gold, Teflon, stainless steel, paper, and aluminum foil as substrates for the aza-Michael addition. (Highly hydrophilic surfaces like glass could not be used because of the extent to which the solvent acetonitrile spreads, making collection of product from the surface particularly difficult). Again, all surfaces provided the expected reaction product, indicating the lack of chemical effects, except for the differences in efficiency of the surface reaction. For example, more products were collected from the inert gold surface than from Teflon (see Figure S2, Supporting Information for detail). Different surfaces yielded products at distinctive rates because hydrophilic surfaces such as gold produced larger surface areas than Teflon, which in turn afforded faster solvent evaporation and, eventually, reagent concentration and more effective collisions in a unit time.

Aside from the relevance of the drop-casting method in synthesis, it also has some inherent advantages in analytical mass spectrometry. For example, the acrylamide and benzaldehyde reactants used in the aza-Michael addition (as Michael acceptor) and the Mannich reaction (as nonenolizable carbonyl compound) are not effectively ionized by ESI (suppose the goal was to specifically analyze them by ESI-MS). However, through chemical derivatization, such compounds can be converted into forms that are easily ionized by ESI [31,32]. A rapid method of chemical derivatization is thus desirable in order not to significantly increase the ESI-MS analysis time. Unfortunately, traditional derivatization via wet chemistry often involves harsh conditions (e.g., high temperatures and acidic media), with long reaction times. The drop-casting conditions have proven to be useful in providing specific derivatives in a matter of a few minutes (Figures 1, 2, and Table 1). Even more interesting is the ability to analyze the nominally dry reaction mixture (after drop-casting) directly from the surface using a surface analysis technique such as DESI, without dissolving it into solution. This capability was

\section{(a) [PRODUCT+H]+}
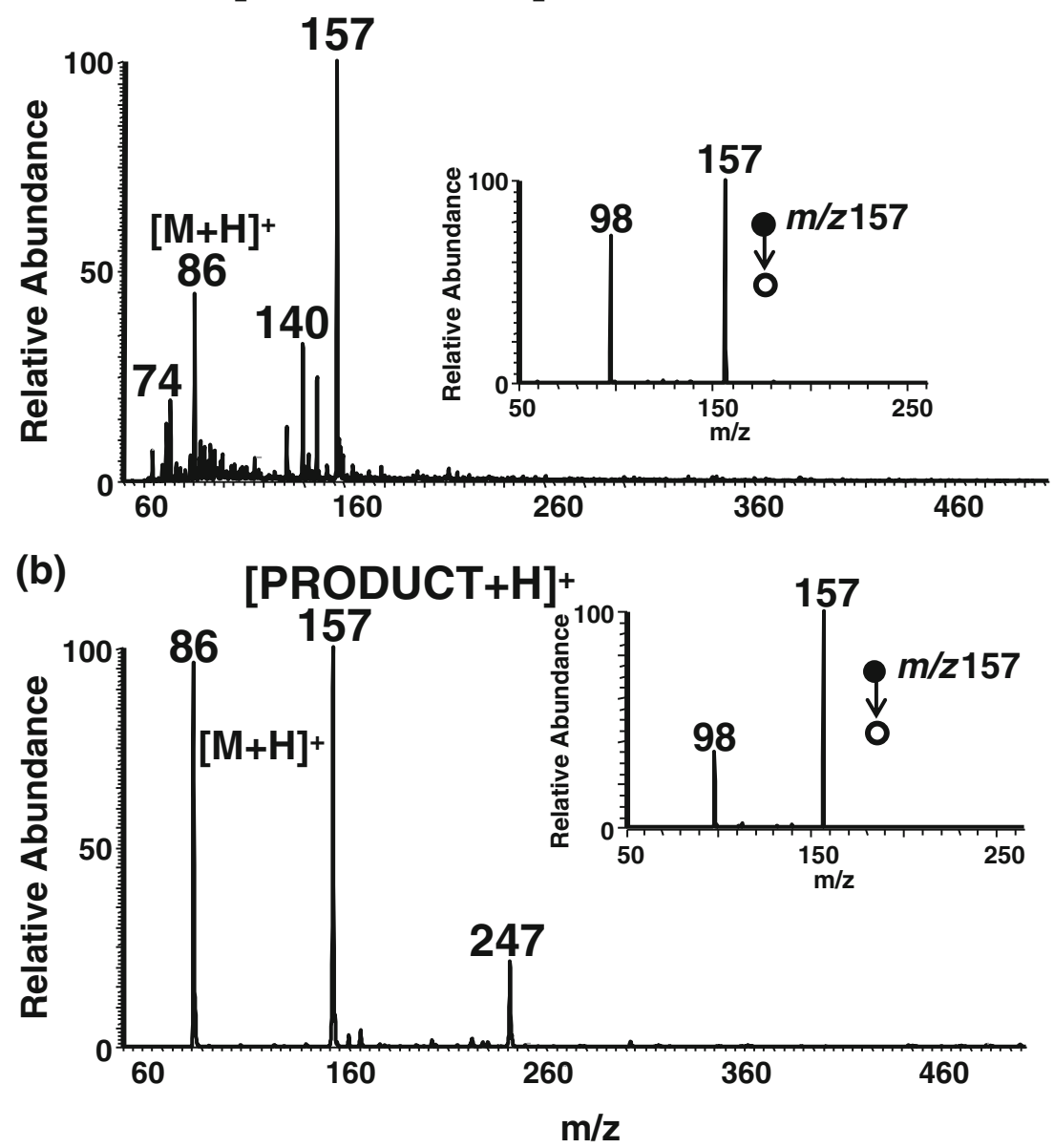

Figure 4. (a) Desorption electrospray ionization (DESI)-MS analysis of drop-casting products directly from Teflon surface: $2 \mu \mathrm{L}$ each of acrylamide $(28 \mathrm{mM})$ and piperidine $(\mathrm{M}, 33 \mathrm{mM})$ solutions in acetonitrile were deposited at the surface and 6 min was allowed for mixture to dry before DESI analysis using methanol/water (1:1, vol/vol) spray solvent; (b) reactive DESI using $\sim 0.4 \mathrm{mM}$ piperidine in methanol/water $(1: 1, \mathrm{vol} / \mathrm{vol})$ solution as DESI spray directed at dry acrylamide $(4 \mu \mathrm{g}$ absolute amount). Inserts in (a) and (b) indicate MS/MS product ion spectra at $\mathrm{m} / \mathrm{z} 157$ 
demonstrated by using DESI to analyze the aza-Michael addition product directly from Teflon surface after dropcasting $2 \mu \mathrm{L}$ each of acrylamide and piperidine solutions in acetonitrile onto the Teflon surface. The resulting spot was dried in the open air for $6 \mathrm{~min}$, and the DESI-MS recorded using $\mathrm{MeOH} / \mathrm{H}_{2} \mathrm{O}(1: 1$, vol/vol $)$ spray solvent is as shown in Figure 4a. The product was detected at $\mathrm{m} / \mathrm{z} 157$ and its identity was confirmed via CID. Unreacted piperidine was also detected at $m / z$ 86. Analysis of Mannich reaction products directly from the reaction surface using DESI was also achieved, and the result is provided in (Figure S3, Supporting Information). It is also recognized that with DESI, no derivatization step may be needed in the MS analysis; it can be operated in a reactive mode (i.e., reactive DESI $[10,11]$ ) in which the sample to be analyzed (e.g., acrylamide) is placed on the surface and then directly analyzed with a DESI spray solvent containing a reactive reagent (e.g., piperidine). In this case, derivatization occurs in-situ during the DESI analysis at ambient conditions. Figure $4 \mathrm{~b}$ shows the result of reactive DESI analysis of acrylamide using piperidine solution $(0.4 \mathrm{mM})$ in $\mathrm{MeOH} /$ $\mathrm{H}_{2} \mathrm{O}(1: 1, \mathrm{vol} / \mathrm{vol})$ as the DESI spray solvent. Here too, the derivatized product is observed in the MS at $m / z 157$.

\section{Conclusions}

An interfacial version of the aza-Michael addition has been achieved through drop-casting, which yielded rate enhancement over the conventional bulk solution-phase reactions performed on the same scale with no added catalyst. The increase in reaction yield is attributed to reagent concentration in the thin film as solvent evaporates. The expected reaction products were collected from all types of surfaces used, including inert gold substrates and others such as stainless steel and aluminum foil, indicating no apparent chemical surface effects. However, the physical properties of the surfaces employed were found to be important as higher reaction efficiencies were achieved on surfaces that allowed rapid solvent evaporation. A more efficient form of indirect Mannich reaction was also achieved at ambient surfaces through the use of the drop-casting procedure. This thin film method may be a simple, efficient, and practical method for preparing reaction products or intermediates for use in the synthesis of diverse chemical species, including various amino ketones. It is, however, recommended that this protocol should be used for small scale chemical synthesis, a condition in which evaporation of large volumes of organic solvents can be avoided. The drop-casting method may also advance sample analysis via MS by allowing rapid and effective sample derivatization compared with wet chemistry. Direct analysis of the surface product is also achievable using desorption electrospray ionization and (presumably) by other ambient ionization techniques. Moreover, the accelerated reaction products can be collected from the surface for subsequent use, or analysis by other analytical techniques such as nuclear magnetic resonance.

\section{Acknowledgment}

The authors acknowledge funding for this work by the National Science Foundation (CHE NSF 0848650 and 0852740).

\section{References}

1. Girod, M., Moyano, E., Campbell, D.I., Cooks, R.G.: Accelerated bimolecular reactions in microdroplets studied by desorption electrospray ionization mass spectrometry. Chem. Sci. 2, 501-510 (2011)

2. Reboule, I., Gil, R., Collin, J.: Aza-Michael Reactions Catalyzed by Samarium Diiodide. Tetrahedron Lett. 46, 7761-7764 (2005)

3. Ambhaikar, N.B., Snyder, J.P., Liotta, D.C.: Diastereoselective addition of chlorotitanium enolate of N-Acyl thiazolidinethione to O-Methyl oximes: a novel, stereoselective synthesis of $\alpha, \beta$-Disubstituted $\beta$-Amino Carbonyl Compounds via Chiral auxiliary mediated azetine formation. J. Am. Chem. Soc. 125, 3690 (2003)

4. Loh, T.-P., Wei, L.L.: Indium trichloride-catalyzed conjugate addition of amines to $\alpha, \beta$-Ethylenic compounds in water. Synlett 9, 975-976 (1998)

5. Bartoli, G., Bartolacci, M., Giuliani, A., Marcantoni, E., Massimo, M., Torregiani, E.: Improved heteroatom nucleophilic addition to electronpoor alkenes promoted by $\mathrm{CeCl}_{3} \cdot 7 \mathrm{H}_{2} \mathrm{O} / \mathrm{NaI}$ system supported on alumina in solvent-free conditions. J. Org. Chem. 70, 169-175 (2005)

6. Jenner, G.: Catalytic high pressure synthesis of hindered $\beta$-Aminoesters. Tetrahedron Lett. 36, 233-236 (1995)

7. Duan, Z., Xuan, X., Li, T., Yang, C., Wu, Y.: Cerium (IV) ammonium nitrate (CAN) catalyzed aza-michael addition of amines to $\alpha, \beta$ unsaturated electrophiles. Tetrahedron Lett. 47, 5433-5436 (2006)

8. Varala, R., Sreelatha, N., Adapa, S.R.: Ceric ammonium nitrate catalyzed aza-michael addition of aliphatic amines to a, b-Unsaturated carbonyl compounds and nitriles in water. Synlett 10, 1549-1553 (2006)

9. Kang, Q., Zhang, Y.: N-Heterocyclic carbene-catalyzed aza-Michael Addition. Org. Biomol. Chem. 9, 6715 (2011)

10. Miao, Z., Chen, H.: Direct analysis of liquid samples by Desorption Electrospray Ionization-Mass Spectrometry (DESI-MS). J. Am. Soc. Mass Spectrom. 20, 10-19 (2009)

11. Barbara, E.J., Eyler, R.J., Powell, H.D.: Reactive desorption electrospray ionization for rapid screening of guests for supramolecular inclusion complexes. Rapid Commun. Mass Spectrom. 22, 4121-4128 (2008)

12. Badu-Tawiah, A.K., Campbell, I.D., Cooks, R.G.: Reactions of microsolvated organic compounds at ambient surfaces: droplet velocity, charge state and solvent effects. J. Am. Soc. Mass Spectrom (2012). doi:10.1007/s13361-012-0365-3

13. Badu-Tawiah, A.K., Wu, C., Cooks, R.G.: Ambient ion soft landing. Anal. Chem. 83, 2648-2654 (2011)

14. Badu-Tawiah, A.K., Cyriac, J., Cooks, R.G.: Reactions of organic ions at ambient surfaces in a solvent-free environment. J. Am. Soc. Mass Spectrom (2012). doi:10.1007/s13361-012-0337-7

15. Song, H., Chen, L.D., Ismagilov, F.R.: Reactions in droplets in microfluidic channels. Angew. Chem. Int. Ed. 45, 7336-7356 (2006)

16. Jorabchi, K., Smith, L.M.: Single droplet separations and surface partition coefficient measurements using laser ablation mass spectrometry. Anal. Chem. 81(23), 9682-9688 (2009)

17. Ablyazov, P.N., Vasilevskaya, V.V., Khokhlov, A.R.: Reactions in surface microreactors: computer simulation. Colloid J. 69(3), 265-271 (2007)

18. Cech, N.B., Enke, C.G.: Relating electrospray ionization response to nonpolar character of small peptides. Anal. Chem. 72, 2717-2723 (2000)

19. Girod, M., Dagany, X., Antoine, R.: Relation between charge state distributions of peptide anions and $\mathrm{pH}$ changes in the electrospray plume. A mass spectrometry and optical spectroscopy investigation. Int. J. Mass Spectrom. 308, 41-48 (2011)

20. Orita, A., Uehara, G., Miwa, K., Otera, J.: Rate Acceleration of Organic Reaction by Immediate Solvent Evaporation. Chem. Commun. 4729 4731 (2006)

21. Fenn, J.B., Mann, M., Meng, C.K., Wong, S.F., Whitehouse, C.M.P.: Electrospray ionization for mass spectrometry of large biomolecules. Science 246(4926), 64-71 (1989) 
22. Cole, R.B.: Electrospray Ionization Mass Spectrometry: Fundamentals Instrumentation \& Applications, 1st edn. John Wiley \& Sons, New York (1997)

23. Trost, B.M., Terrell, L.R.: A direct catalytic asymmetric mannich-type reaction to syn-amino alcohols. J. Am. Chem. Soc. 125(1), 338-339 (2003)

24. Matsunaga, S., Kumagai, N., Harada, S., Shibasaki, M.: Anti-selective direct catalytic asymmetric mannich-type reaction of hydroxyketone providing $\beta$-amino alcohols. J. Am. Chem. Soc. 125(16), 4712-4713 (2003)

25. Kobayashi, S., Hamada, T., Manabe, K.: The catalytic asymmetric mannich-type reactions in aqueous media. J. Am. Chem. Soc. 124(20), 5640-5641 (2002)

26. List, B.: The direct catalytic asymmetric three-component mannich reaction. J. Am. Chem. Soc. 122, 9336-9337 (2000)

27. Hayashi, Y., Tsuboi, W., Ashimine, I., Urushima, T., Shoji, M., Sakai, K.: The direct and enantioselective, one-pot, three-component. cross-mannich reaction of aldehydes. Angew. Chem. Int. Ed. 42, 3677-3680 (2003)
28. Ohta, K., Morokuma, K.: An MO study of SN2 reactions in hydrated gas clusters: Hydrated Hydroxide $\left[\left(\mathrm{H}_{2} \mathrm{O}\right) \mathrm{nOH}-\right]+$ Hydrated Methyl Chloride $[\mathrm{MeCl}(\mathrm{H} 2 \mathrm{O}) \mathrm{m}]$.fwdarw. Methanol + Chloride $+(\mathrm{n}+\mathrm{m})$ Water. J. Phys. Chem 89, 5845 (1985)

29. Bohme, D.K., Raksit, A.B.: Gas-Phase measurements of the influence of stepwise solvation on the kinetics of nucleophilic displacement reactions with chloromethane and bromomethane at room temperature. J. Am. Chem. Soc. 106, 3447 (1984)

30. Jonathan, N.: The infrared and raman spectra and structure of acrylamide. J. Mol. Spectrosc. 6, 205-214 (1961)

31. Naven, T.J.P., Harvey, D.J.: Cationic derivatization of oligosaccharides with Girard's T reagent for improved performance in matrix-assisted laser desorption/ionization and electrospray mass spectrometry. Rapid Commun. Mass Spectrom. 10, 829-834 (1996)

32. Eggink, M., Wijtmans, M., Ekkebus, R., Lingeman, H., de Esch, I.J.P., Kool, J., Niessen, W.M.A., Irth, H.: Development of a selective ESI-MS derivatization reagent: synthesis and optimization for the analysis of aldehydes in biological mixtures. Anal. Chem. 80, 9042-9051 (2008) 